\title{
Gravity inequalities and the mean temperature on a planet
}

\author{
Jia Jin Wen ${ }^{1}$, Jun Yuan ${ }^{2^{*}}$, Shan He Wu ${ }^{3}$ and Tian Yong Han ${ }^{1}$
}

\author{
"Correspondence: \\ yuanjun_math@126.com \\ ${ }^{2}$ School of Information Engineering, \\ Nanjing Xiaozhuang University, \\ Nanjing, Jiangsu 211171, P.R. China \\ Full list of author information is \\ available at the end of the article
}

$$
\begin{aligned}
& \text { Abstract } \\
& \text { In the centered surround system } S^{(2)}\{P, \Gamma\} \text {, we establish the following gravity } \\
& \text { inequalities: } \\
& \qquad\left(1+\frac{5}{2 \pi} \frac{\mathrm{e}^{2}}{1-\mathrm{e}^{2}}\right)\left(\frac{2 \pi}{|\Gamma|}\right)^{2} \leq \frac{1}{|\Gamma|} \oint_{\Gamma} \frac{1}{\|A-P\|^{2}} \leq\left(1+\frac{16-\pi}{4 \pi} \frac{\mathrm{e}^{2}}{1-\mathrm{e}^{2}}\right)\left(\frac{2 \pi}{|\Gamma|}\right)^{2}, \\
& \text { where } \Gamma \text { is an ellipse, } P \text { and e are one of the foci and the eccentricity of the ellipse, } \\
& \text { respectively, and } A \in \Gamma \text { is a satellite of the centered surround system } S^{(2)}\{P, \Gamma\} \text {. We } \\
& \text { also demonstrate the applications of the inequalities in the temperature research, and } \\
& \text { we obtain an approximate mean temperature formula as follows: } \\
& \qquad \bar{T} \approx\left(1+0.9095071300973198 \ldots \times \frac{\mathrm{e}^{2}}{1-\mathrm{e}^{2}}\right)\left(\frac{2 \pi}{|\Gamma|}\right)^{2}, \quad \forall \mathrm{e} \in(0,1),
\end{aligned}
$$

where the $\bar{T}$ is the mean temperature on a planet.

MSC: 26D15; 26E60; 51K05; 52A40

Keywords: centered surround system; mean gravity norm; isoperimetric inequality; Newton formula; Euler formula

\section{Introduction}

It is well known that there are eight planets in the solar system, i.e., Mercury, Venus, Earth, Mars, Jupiter, Saturn, Uranus, and Neptune. In space science, we are concerned with the radiation energy of the Sun since it will directly affect our daily life. Since the temperature on a planet is dependent on the radiation energy and the radiation energy is related to the gravity of the Sun, we are especially interested in the gravity norm of the Sun.

In space science, we need to know the mean temperature on a planet. Unfortunately, it is very difficult to measure the mean temperature on a planet. Therefore, it is of theoretical significance to study the mean temperature on a planet by means of mathematics.

At present, the research of temperature on the Earth is a topic of focus in the world. In our daily life, we are concerned with temperature change, which will directly affect our daily life. Since the rain and the air humidity are related to the temperature and our daily life is dependent on the rain and the air humidity, it is of value in applications to study the mean temperature on the Earth.

(c) 2016 Wen et al. This article is distributed under the terms of the Creative Commons Attribution 4.0 International License (http://creativecommons.org/licenses/by/4.0/), which permits unrestricted use, distribution, and reproduction in any medium, provided you give appropriate credit to the original author(s) and the source, provide a link to the Creative Commons license, and indicate if changes were made. 
In this paper, our motivation is to study the mean temperature on a planet by means of the theory of mean [1]. To this end, we first introduce the basic concepts in the surround system [2-5], and we illustrate the background and the significance of these concepts in space science. Next, we establish several identities and inequalities involving the centered surround system $S^{(2)}\{P, \Gamma\}$, in particular, the mean gravity norm formula, as well as we illustrate the coefficients in these inequalities are the best constants. Next, we prove gravity inequalities in the centered surround system $S^{(2)}\{P, \Gamma\}$, which are also isoperimetric-type inequalities $[6,7]$. Finally, we demonstrate the applications of our results in the temperature research on a planet, and we obtain an approximate mean temperature formula.

\section{Basic concepts and main result}

In $[2-5,8]$, the authors systematically studied the theory of satellite and obtained some results which have the application value. But in space science, the centered surround system $S^{(2)}\{P, \Gamma\}[2-5]$ has its special properties, that is, where the $\Gamma$ is an ellipse and $P$ is one of the foci of the ellipse [8]. Therefore, it is necessary for us to do further research on this centered surround system.

Let the particle $A \in \mathbb{R}^{2}$ be regarded as the Earth, and let its motion trajectory be the ellipse

$$
\Gamma \triangleq\left\{x \mathbf{i}+y \mathbf{j} \in \mathbb{R}^{2} \mid \frac{x^{2}}{a^{2}}+\frac{y^{2}}{b^{2}}=1, x, y \in \mathbb{R}, a \geq b>0\right\},
$$

where $\mathbf{i}=(1,0), \mathbf{j}=(0,1), \mathbb{R} \triangleq(-\infty, \infty), \mathbb{R}^{2} \triangleq \mathbb{R} \times \mathbb{R}$, and let the particle $P \triangleq-\sqrt{a^{2}-b^{2}} \mathbf{i}$ be regarded as the Sun, which is a focus of the ellipse $\Gamma$. Then the set $S^{(2)}\{P, \Gamma\} \triangleq\{P, \Gamma\}$ is a centered surround system [2-5].

We remark here that the foci of the ellipse $\Gamma$ are $-c \mathbf{i}$ and $c \mathbf{i}$, where $c=\sqrt{a^{2}-b^{2}} \geq 0$, and the eccentricity of the ellipse $\Gamma$ is $\mathrm{e} \triangleq c / a \in[0,1)$. Note that the e in this paper is the eccentricity of the ellipse $\Gamma$ rather than the Euler constant $e$, that is,

$$
\mathrm{e} \neq e \triangleq \lim _{n \rightarrow \infty}\left(1+\frac{1}{n}\right)^{n}=2.718281828459045 \ldots
$$

Let the masses of the Earth $A$ and the Sun $P$ be $m>0$ and $M>0$, respectively. Then, according to the law of universal gravitation, the gravity of the Earth $A$ to the $\operatorname{Sun} P$ is

$$
\mathbf{F}(A, P)=\frac{\operatorname{GmM}(A-P)}{\|A-P\|^{3}}
$$

where the $G$ is the gravitational constant in the solar system. Without loss of generality, here we assume that $G m M=1$.

We say that

$$
\|\mathbf{F}(A, P)\|=\frac{1}{\|A-P\|^{2}} \quad \text { and } \quad \overline{\|\mathbf{F}(A, P)\|} \triangleq \frac{1}{|\Gamma|} \oint_{\Gamma} \frac{1}{\|A-P\|^{2}}
$$

are the gravity norm and the mean gravity norm of the gravity $\mathbf{F}(A, P)$, respectively $[5,9,10]$.

In general, the mean gravity norm $\overline{\|\mathbf{F}(A, P)\|}$ cannot be expressed by the elementary functions since it involves the elliptic integral [11]. Therefore, in order to facilitate the 
applications, we need to find its sharp lower and upper bounds, which can be expressed by the elementary functions.

In this paper, our main result is as follows.

Theorem 1 (Gravity inequalities) Let $S^{(2)}\{P, \Gamma\}$ be a centered surround system, where the $\Gamma$ is an ellipse and the $P$ is one of the foci of the ellipse. Then we have the following isoperimetric-type inequalities [6, 7]:

$$
\left(1+\frac{5}{2 \pi} \frac{\mathrm{e}^{2}}{1-\mathrm{e}^{2}}\right)\left(\frac{2 \pi}{|\Gamma|}\right)^{2} \leq \overline{\|\mathbf{F}(A, P)\|} \leq\left(1+\frac{16-\pi}{4 \pi} \frac{\mathrm{e}^{2}}{1-\mathrm{e}^{2}}\right)\left(\frac{2 \pi}{|\Gamma|}\right)^{2},
$$

where the e is the eccentricity of the ellipse. The equalities in (2) hold if and only if $\Gamma$ is a circle.

In [5], the authors obtained the following isoperimetric inequality [6, 7] (see (66) in [5]):

$$
\overline{\|\mathbf{F}(A, P)\|} \geq\left(\frac{2 \pi}{|\Gamma|}\right)^{2}
$$

where the $\Gamma$ is a smooth and convex Jordan closed curve in $\mathbb{R}^{2}[2-5,12]$. Obviously, inequalities (2) are both an improvement and an expansion of inequality (3).

In space science, the orbit $\Gamma$ of a satellite $A$ is approximate to a circle [8], that is, the eccentricity e of the ellipse $\Gamma$ is very small. Since the error

$$
\frac{16-\pi}{4 \pi}-\frac{5}{2 \pi}=0.22746482927568604 \ldots
$$

of the coefficients $(16-\pi) /(4 \pi)$ and $5 /(2 \pi)$ is not very large and the eccentricity e is very small, we see that inequalities (2) are sharp.

We remark here that, by the isoperimetric inequalities [5]

$$
\pi a b=\operatorname{Area} D(\Gamma) \leq \frac{|\Gamma|^{2}}{4 \pi}
$$

and the $p$-mean inequality $[1,5,9,13-16]$

$$
\begin{aligned}
\frac{|\Gamma|}{2 \pi} & =\frac{1}{2 \pi} \int_{0}^{2 \pi} \sqrt{a^{2} \sin ^{2} \theta+b^{2} \cos ^{2} \theta} \mathrm{d} \theta \\
& \leq \sqrt{\frac{1}{2 \pi} \int_{0}^{2 \pi}\left(a^{2} \sin ^{2} \theta+b^{2} \cos ^{2} \theta\right) \mathrm{d} \theta} \\
& =\sqrt{\frac{a^{2}+b^{2}}{2}},
\end{aligned}
$$

we have

$$
\sqrt{a b} \leq \frac{|\Gamma|}{2 \pi} \leq \sqrt{\frac{a^{2}+b^{2}}{2}}
$$

Therefore, the $|\Gamma| /(2 \pi)$ is a mean of the positive real numbers $a$ and $b$. 


\section{Preliminaries}

In order to prove Theorem 1, we need to establish several identities and inequalities as follows.

According to the theory of mathematical analysis, we have Lemma 1.

Lemma 1 Let $f: \mathbb{R} \rightarrow \mathbb{R}$ be a periodic function with the period $T$. Then we have

$$
\int_{t}^{T+t} f(x) \mathrm{d} x=\int_{0}^{T} f(x) \mathrm{d} x, \quad \forall t \in \mathbb{R}
$$

Lemma 2 For any continuous function $g:[0,1] \rightarrow \mathbb{R}$, we have

$$
\frac{1}{2 \pi} \int_{0}^{2 \pi} g\left(\cos ^{2} \theta\right) \mathrm{d} \theta=\frac{2}{\pi} \int_{0}^{\frac{\pi}{2}} g\left(\cos ^{2} \theta\right) \mathrm{d} \theta .
$$

Proof Since the function $g\left(\cos ^{2} \theta\right)$ is both an even function and a periodic function with the period $\pi$, according to Lemma 1 , we have

$$
\begin{aligned}
\frac{1}{2 \pi} \int_{0}^{2 \pi} g\left(\cos ^{2} \theta\right) \mathrm{d} \theta & =\frac{1}{2 \pi} \int_{-\pi}^{\pi} g\left(\cos ^{2} \theta\right) \mathrm{d} \theta \\
& =\frac{1}{\pi} \int_{0}^{\pi} g\left(\cos ^{2} \theta\right) \mathrm{d} \theta \\
& =\frac{1}{\pi} \int_{-\frac{\pi}{2}}^{\frac{\pi}{2}} g\left(\cos ^{2} \theta\right) \mathrm{d} \theta \\
& =\frac{2}{\pi} \int_{0}^{\frac{\pi}{2}} g\left(\cos ^{2} \theta\right) \mathrm{d} \theta .
\end{aligned}
$$

This ends the proof of Lemma 2.

Lemma 3 (Mean gravity norm formula) Under the hypotheses in Theorem 1, we have

$$
\overline{\|\mathbf{F}(A, P)\|}=\left(\frac{2 \pi}{|\Gamma|}\right)^{2}\left(\frac{2}{\pi} \int_{0}^{\frac{\pi}{2}} \sqrt{1-\mathrm{e}^{2} \cos ^{2} \theta} \mathrm{d} \theta\right)\left[\frac{2}{\pi} \int_{0}^{\frac{\pi}{2}} \frac{1+\mathrm{e}^{2} \cos ^{2} \theta}{\left(1-\mathrm{e}^{2} \cos ^{2} \theta\right)^{3 / 2}} \mathrm{~d} \theta\right] .
$$

Proof Since $\Gamma=\left\{x \mathbf{i}+y \mathbf{j} \in \mathbb{R}^{2} \mid x=a \cos \theta, y=b \sin \theta, \theta \in[0,2 \pi]\right\}$, we have

$$
\begin{aligned}
\|A-P\| & =\sqrt{(a \cos \theta+c)^{2}+(b \sin \theta)^{2}} \\
& =\sqrt{a^{2} \cos ^{2} \theta+2 a c \cos \theta+c^{2}+\left(a^{2}-c^{2}\right) \sin ^{2} \theta} \\
& =a+c \cos \theta=a(1+\mathrm{e} \cos \theta),
\end{aligned}
$$

that is,

$$
\|A-P\|=a(1+\mathrm{e} \cos \theta)
$$

Since

$$
\frac{\mathrm{d} s}{\mathrm{~d} \theta}=\sqrt{\left(\frac{\mathrm{d} x}{\mathrm{~d} \theta}\right)^{2}+\left(\frac{\mathrm{d} y}{\mathrm{~d} \theta}\right)^{2}}=a \sqrt{1-\mathrm{e}^{2} \cos ^{2} \theta},
$$


by (8) and (9), we have

$$
\begin{aligned}
& \overline{\|\mathbf{F}(A, P)\|}=\frac{1}{|\Gamma|} \oint_{\Gamma} \frac{1}{\|A-P\|^{2}} \mathrm{~d} s \\
& =\frac{1}{|\Gamma|} \int_{0}^{2 \pi} \frac{1}{a^{2}(1+\mathrm{e} \cos \theta)^{2}} \sqrt{\left(\frac{\mathrm{d} x}{\mathrm{~d} \theta}\right)^{2}+\left(\frac{\mathrm{d} y}{\mathrm{~d} \theta}\right)^{2}} \mathrm{~d} \theta \\
& =\frac{1}{|\Gamma|} \int_{0}^{2 \pi} \frac{a \sqrt{1-\mathrm{e}^{2} \cos ^{2} \theta}}{a^{2}(1+\mathrm{e} \cos \theta)^{2}} \mathrm{~d} \theta \\
& =\frac{1}{a|\Gamma|} \int_{0}^{2 \pi} \frac{\sqrt{1-\mathrm{e}^{2} \cos ^{2} \theta}}{(1+\mathrm{e} \cos \theta)^{2}} \mathrm{~d} \theta \\
& =\frac{1}{a|\Gamma|} \int_{0}^{2 \pi} \frac{\sqrt{1-\mathrm{e} \cos \theta}}{(1+\mathrm{e} \cos \theta)^{3 / 2}} \mathrm{~d} \theta,
\end{aligned}
$$

that is,

$$
\overline{\|\mathbf{F}(A, P)\|}=\frac{1}{a|\Gamma|} \int_{0}^{2 \pi} \frac{\sqrt{1-\mathrm{e} \cos \theta}}{(1+\mathrm{e} \cos \theta)^{3 / 2}} \mathrm{~d} \theta
$$

In (10), set $\theta=\vartheta-\pi$. Since the cosine function $\cos x$ is a periodic function with the period $2 \pi$, by Lemma 1 , we have

$$
\begin{aligned}
\overline{\|\mathbf{F}(A, P)\|} & =\frac{1}{a|\Gamma|} \int_{0}^{2 \pi} \frac{\sqrt{1-\mathrm{e} \cos \theta}}{(1+\mathrm{e} \cos \theta)^{3 / 2}} \mathrm{~d} \theta \\
& =\frac{1}{a|\Gamma|} \int_{\pi}^{2 \pi+\pi} \frac{\sqrt{1-\mathrm{e} \cos (\vartheta-\pi)}}{[1+\mathrm{e} \cos (\vartheta-\pi)]^{3 / 2}} \mathrm{~d}(\vartheta-\pi) \\
& =\frac{1}{a|\Gamma|} \int_{\pi}^{2 \pi+\pi} \frac{\sqrt{1+\mathrm{e} \cos \vartheta}}{(1-\mathrm{e} \cos \vartheta)^{3 / 2}} \mathrm{~d} \vartheta \\
& =\frac{1}{a|\Gamma|} \int_{0}^{2 \pi} \frac{\sqrt{1+\mathrm{e} \cos \vartheta}}{(1-\mathrm{e} \cos \vartheta)^{3 / 2}} \mathrm{~d} \vartheta \\
& =\frac{1}{a|\Gamma|} \int_{0}^{2 \pi} \frac{\sqrt{1+\mathrm{e} \cos \theta}}{(1-\mathrm{e} \cos \theta)^{3 / 2}} \mathrm{~d} \theta,
\end{aligned}
$$

that is,

$$
\overline{\|\mathbf{F}(A, P)\|}=\frac{1}{a|\Gamma|} \int_{0}^{2 \pi} \frac{\sqrt{1+\mathrm{e} \cos \theta}}{(1-\mathrm{e} \cos \theta)^{3 / 2}} \mathrm{~d} \theta
$$

By (10) and (11), we get

$$
\begin{aligned}
\overline{\|\mathbf{F}(A, P)\|} & =\frac{1}{a|\Gamma|} \int_{0}^{2 \pi} \frac{1}{2}\left[\frac{\sqrt{1-\mathrm{e} \cos \theta}}{(1+\mathrm{e} \cos \theta)^{3 / 2}}+\frac{\sqrt{1+\mathrm{e} \cos \theta}}{(1-\mathrm{e} \cos \theta)^{3 / 2}}\right] \mathrm{d} \theta \\
& =\frac{1}{a|\Gamma|} \int_{0}^{2 \pi} \frac{(1-\mathrm{e} \cos \theta)^{2}+(1+\mathrm{e} \cos \theta)^{2}}{2\left(1-\mathrm{e}^{2} \cos ^{2} \theta\right)^{3 / 2}} \mathrm{~d} \theta \\
& =\frac{1}{a|\Gamma|} \int_{0}^{2 \pi} \frac{1+\mathrm{e}^{2} \cos ^{2} \theta}{\left(1-\mathrm{e}^{2} \cos ^{2} \theta\right)^{3 / 2}} \mathrm{~d} \theta,
\end{aligned}
$$


that is,

$$
\overline{\|\mathbf{F}(A, P)\|}=\frac{1}{a|\Gamma|} \int_{0}^{2 \pi} \frac{1+\mathrm{e}^{2} \cos ^{2} \theta}{\left(1-\mathrm{e}^{2} \cos ^{2} \theta\right)^{3 / 2}} \mathrm{~d} \theta .
$$

On the other hand, by (9), we have

$$
|\Gamma|=\int_{0}^{2 \pi} \sqrt{\left(\frac{\mathrm{d} x}{\mathrm{~d} \theta}\right)^{2}+\left(\frac{\mathrm{d} y}{\mathrm{~d} \theta}\right)^{2}} \mathrm{~d} \theta=a \int_{0}^{2 \pi} \sqrt{1-\mathrm{e}^{2} \cos ^{2} \theta} \mathrm{d} \theta .
$$

According to (12), (13), and Lemma 2, we get

$$
\begin{aligned}
\overline{\|\mathbf{F}(A, P)\|} & =\frac{1}{a|\Gamma|} \int_{0}^{2 \pi} \frac{1+\mathrm{e}^{2} \cos ^{2} \theta}{\left(1-\mathrm{e}^{2} \cos ^{2} \theta\right)^{3 / 2}} \mathrm{~d} \theta \\
& =\frac{1}{|\Gamma|^{2}} \int_{0}^{2 \pi} \sqrt{1-\mathrm{e}^{2} \cos ^{2} \theta} \mathrm{d} \theta \int_{0}^{2 \pi} \frac{1+\mathrm{e}^{2} \cos ^{2} \theta}{\left(1-\mathrm{e}^{2} \cos ^{2} \theta\right)^{3 / 2}} \mathrm{~d} \theta \\
& =\left(\frac{2 \pi}{|\Gamma|}\right)^{2}\left(\frac{2}{\pi} \int_{0}^{\frac{\pi}{2}} \sqrt{1-\mathrm{e}^{2} \cos ^{2} \theta} \mathrm{d} \theta\right)\left[\frac{2}{\pi} \int_{0}^{\frac{\pi}{2}} \frac{1+\mathrm{e}^{2} \cos ^{2} \theta}{\left(1-\mathrm{e}^{2} \cos ^{2} \theta\right)^{3 / 2}} \mathrm{~d} \theta\right] .
\end{aligned}
$$

That is, equation (7) is proved. The proof of Lemma 3 is completed.

Lemma 4 The inequality

$$
\frac{2}{\pi} \int_{0}^{\frac{\pi}{2}} \sqrt{1-\mathrm{e}^{2} \cos ^{2} \theta} \mathrm{d} \theta \geq 1-\delta \mathrm{e}^{2}
$$

holds if and only if

$$
\delta \geq 1-\frac{2}{\pi}
$$

where the $\delta$ is a positive constant.

Proof Assume that (15) holds, we prove that (14) holds as follows.

By $0 \leq \mathrm{e}<1,0 \leq \mathrm{e}^{2} \cos ^{2} \theta<1$ and the Newton formula

$$
(1+x)^{\mu}=1+\sum_{n=1}^{\infty} \frac{1}{n !} \prod_{k=1}^{n}(\mu+1-k) x^{n}, \quad \forall x \in(-1,1),
$$

we get

$$
\begin{aligned}
\sqrt{1-\mathrm{e}^{2} \cos ^{2} \theta} & =1+\sum_{n=1}^{\infty} \frac{1}{n !} \prod_{k=1}^{n}\left(\frac{1}{2}+1-k\right)\left(-\mathrm{e}^{2} \cos ^{2} \theta\right)^{n} \\
& =1-\mathrm{e}^{2} \sum_{n=1}^{\infty} \frac{(2 n-1) ! !}{(2 n-1)(2 n) ! !} \mathrm{e}^{2(n-1)} \cos ^{2 n} \theta
\end{aligned}
$$

that is,

$$
\sqrt{1-\mathrm{e}^{2} \cos ^{2} \theta}=1-\mathrm{e}^{2} \sum_{n=1}^{\infty} \frac{(2 n-1) ! !}{(2 n-1)(2 n) ! !} \mathrm{e}^{2(n-1)} \cos ^{2 n} \theta,
$$


where

$$
(2 n) ! ! \triangleq 2 \times 4 \times 6 \times \cdots \times(2 n) \quad \text { and } \quad(2 n-1) ! ! \triangleq 1 \times 3 \times 5 \times \cdots \times(2 n-1) .
$$

By (17), we get

$$
\frac{2}{\pi} \int_{0}^{\frac{\pi}{2}} \sqrt{1-\mathrm{e}^{2} \cos ^{2} \theta} \mathrm{d} \theta=1-\mathrm{e}^{2} \sum_{n=1}^{\infty} \frac{(2 n-1) ! !}{(2 n-1)(2 n) ! !} \mathrm{e}^{2(n-1)}\left(\frac{2}{\pi} \int_{0}^{\frac{\pi}{2}} \cos ^{2 n} \theta \mathrm{d} \theta\right)
$$

Since

$$
\frac{2}{\pi} \int_{0}^{\frac{\pi}{2}} \sqrt{1-\cos ^{2} \theta} \mathrm{d} \theta=\frac{2}{\pi} \int_{0}^{\frac{\pi}{2}} \sin \theta \mathrm{d} \theta=\frac{2}{\pi}
$$

in (19), letting $\mathrm{e} \rightarrow 1$, we get

$$
\sum_{n=1}^{\infty} \frac{(2 n-1) ! !}{(2 n-1)(2 n) ! !}\left(\frac{2}{\pi} \int_{0}^{\frac{\pi}{2}} \cos ^{2 n} \theta \mathrm{d} \theta\right)=1-\frac{2}{\pi}
$$

From (15), (19), (21), and $0 \leq \mathrm{e}<1$, we get

$$
\begin{aligned}
\frac{2}{\pi} \int_{0}^{\frac{\pi}{2}} \sqrt{1-\mathrm{e}^{2} \cos ^{2} \theta} \mathrm{d} \theta & =1-\mathrm{e}^{2} \sum_{n=1}^{\infty} \frac{(2 n-1) ! !}{(2 n-1)(2 n) ! !} \mathrm{e}^{2(n-1)}\left(\frac{2}{\pi} \int_{0}^{\frac{\pi}{2}} \cos ^{2 n} \theta \mathrm{d} \theta\right) \\
& \geq 1-\mathrm{e}^{2} \sum_{n=1}^{\infty} \frac{(2 n-1) ! !}{(2 n-1)(2 n) ! !}\left(\frac{2}{\pi} \int_{0}^{\frac{\pi}{2}} \cos ^{2 n} \theta \mathrm{d} \theta\right) \\
& =1-\left(1-\frac{2}{\pi}\right) \mathrm{e}^{2} \\
& \geq 1-\delta \mathrm{e}^{2} .
\end{aligned}
$$

That is, inequality (14) is proved.

Next, assume that (14) holds; we prove (15) as follows.

In (14), letting $\mathrm{e} \rightarrow 1$, by (20), we get

$$
\frac{2}{\pi} \geq 1-\delta \quad \Leftrightarrow \quad \delta \geq 1-\frac{2}{\pi}
$$

That is, inequality (15) holds. This proves Lemma 4.

According to the theory of mathematical analysis, we have Lemma 5.

Lemma 5 For any positive integer $n$, we have

$$
\frac{2}{\pi} \int_{0}^{\frac{\pi}{2}} \cos ^{2 n} \theta \mathrm{d} \theta=\frac{(2 n-1) ! !}{(2 n) ! !}
$$

where !! is defined by (18). 


\section{Lemma 6 The inequality}

$$
\frac{2}{\pi} \int_{0}^{\frac{\pi}{2}} \sqrt{1-\mathrm{e}^{2} \cos ^{2} \theta} \mathrm{d} \theta \leq \sqrt{1-\varepsilon \mathrm{e}^{2}}
$$

holds if and only if

$$
\varepsilon \leq \frac{1}{2}
$$

where the $\varepsilon$ is a positive constant.

Proof Assume that (24) holds, we prove (23) holds as follows.

By the $p$-mean inequality $[1,5,9,13-16]$ and Lemma 5 , we see that, for any positive integer $n$, we have

$$
\frac{2}{\pi} \int_{0}^{\frac{\pi}{2}} \cos ^{2 n} \theta \mathrm{d} \theta \geq\left(\frac{2}{\pi} \int_{0}^{\frac{\pi}{2}} \cos ^{2} \theta \mathrm{d} \theta\right)^{n}=\frac{1}{2^{n}} .
$$

In (17), use the following exchanges

$$
\mathrm{e} \leftrightarrow \frac{\mathrm{e}}{\sqrt{2}} \quad \text { and } \quad \theta \leftrightarrow 0,
$$

we get

$$
1-\mathrm{e}^{2} \sum_{n=1}^{\infty} \frac{(2 n-1) ! !}{(2 n-1)(2 n) ! !} \mathrm{e}^{2(n-1)} \frac{1}{2^{n}}=\sqrt{1-\frac{1}{2} \mathrm{e}^{2}} .
$$

From (19), (24), (25), and (26), we get

$$
\begin{aligned}
\frac{2}{\pi} & \int_{0}^{\frac{\pi}{2}} \sqrt{1-\mathrm{e}^{2} \cos ^{2} \theta} \mathrm{d} \theta \\
& =1-\mathrm{e}^{2} \sum_{n=1}^{\infty} \frac{(2 n-1) ! !}{(2 n-1)(2 n) ! !} \mathrm{e}^{2(n-1)}\left(\frac{2}{\pi} \int_{0}^{\frac{\pi}{2}} \cos ^{2 n} \theta \mathrm{d} \theta\right) \\
& \leq 1-\mathrm{e}^{2} \sum_{n=1}^{\infty} \frac{(2 n-1) ! !}{(2 n-1)(2 n) ! !} \mathrm{e}^{2(n-1)} \frac{1}{2^{n}} \\
& =\sqrt{1-\frac{1}{2} \mathrm{e}^{2}} \\
& \leq \sqrt{1-\varepsilon \mathrm{e}^{2}} .
\end{aligned}
$$

Hence inequality (23) is proved.

Next, assume that (23) holds; we prove (24) as follows.

Let $0<\mathrm{e}<1$. By (23), we get

$$
\varepsilon \leq \frac{1}{\mathrm{e}^{2}}\left[1-\left(\frac{2}{\pi} \int_{0}^{\frac{\pi}{2}} \sqrt{1-\mathrm{e}^{2} \cos ^{2} \theta} \mathrm{d} \theta\right)^{2}\right], \quad \forall \mathrm{e} \in(0,1) .
$$


By (27) and Lemma 5, we get

$$
\begin{aligned}
\varepsilon & \leq \lim _{\mathrm{e} \rightarrow 0} \frac{1}{\mathrm{e}^{2}}\left[1-\left(\frac{2}{\pi} \int_{0}^{\frac{\pi}{2}} \sqrt{1-\mathrm{e}^{2} \cos ^{2} \theta} \mathrm{d} \theta\right)^{2}\right] \\
& =\lim _{t \rightarrow 0} \frac{1}{t}\left[1-\left(\frac{2}{\pi} \int_{0}^{\frac{\pi}{2}} \sqrt{1-t \cos ^{2} \theta} \mathrm{d} \theta\right)^{2}\right] \\
& =\lim _{t \rightarrow 0} \frac{\mathrm{d}}{\mathrm{d} t}\left[1-\left(\frac{2}{\pi} \int_{0}^{\frac{\pi}{2}} \sqrt{1-t \cos ^{2} \theta} \mathrm{d} \theta\right)^{2}\right] \\
& =-2 \lim _{t \rightarrow 0}\left(\frac{2}{\pi} \int_{0}^{\frac{\pi}{2}} \sqrt{1-t \cos ^{2} \theta} \mathrm{d} \theta\right) \frac{\mathrm{d}}{\mathrm{d} t}\left(\frac{2}{\pi} \int_{0}^{\frac{\pi}{2}} \sqrt{1-t \cos ^{2} \theta} \mathrm{d} \theta\right) \\
& =-2 \lim _{t \rightarrow 0} \frac{\mathrm{d}}{\mathrm{d} t}\left(\frac{2}{\pi} \int_{0}^{\frac{\pi}{2}} \sqrt{1-t \cos ^{2} \theta} \mathrm{d} \theta\right) \\
& =-2 \lim _{t \rightarrow 0}\left(\frac{2}{\pi} \int_{0}^{\frac{\pi}{2}} \frac{\mathrm{d}}{\mathrm{d} t} \sqrt{1-t \cos ^{2} \theta} \mathrm{d} \theta\right) \\
& =-2 \lim _{t \rightarrow 0}\left(\frac{2}{\pi} \int_{0}^{\frac{\pi}{2}} \frac{-\cos ^{2} \theta}{2 \sqrt{1-t \cos ^{2} \theta}} \mathrm{d} \theta\right) \\
& =\frac{2}{\pi} \int_{0}^{\frac{\pi}{2}} \cos { }^{2} \theta \mathrm{d} \theta \\
& =\frac{1}{2} .
\end{aligned}
$$

Hence inequality (24) holds. The proof of Lemma 6 is completed.

Lemma 7 For any positive integer $n$, we have

$$
\frac{5}{4} \leq \frac{4 n+1}{2 n+1} \prod_{k=1}^{n}\left(1-\frac{1}{4 k^{2}}\right)<\frac{4}{\pi} .
$$

Proof Recall the following famous Euler formula:

$$
\sin x=x \prod_{n=1}^{\infty}\left(1-\frac{x^{2}}{\pi^{2} n^{2}}\right), \quad \forall x \in \mathbb{R} .
$$

In (29), set $x=\pi / 2$, we get

$$
\lim _{n \rightarrow \infty} \prod_{k=1}^{n}\left(1-\frac{1}{4 k^{2}}\right)=\prod_{n=1}^{\infty}\left(1-\frac{1}{4 n^{2}}\right)=\frac{2}{\pi} .
$$

Define an auxiliary sequence $\left\{u_{n}\right\}_{n=1}^{\infty}$ as follows:

$$
\left\{u_{n}\right\}_{n=1}^{\infty}: \quad u_{n} \triangleq \frac{4 n+1}{2 n+1} \prod_{k=1}^{n}\left(1-\frac{1}{4 k^{2}}\right) .
$$

By (30), we have

$$
\lim _{n \rightarrow \infty} u_{n}=\frac{4}{\pi} .
$$


Since

$$
\frac{u_{n+1}}{u_{n}}=\frac{4 n+5}{2 n+3} \times \frac{2 n+1}{4 n+1}\left[1-\frac{1}{4(n+1)^{2}}\right]=\frac{16 n^{3}+36 n^{2}+24 n+5}{16 n^{3}+36 n^{2}+24 n+4}>1 \text {, }
$$

the sequence $\left\{u_{n}\right\}_{n=1}^{\infty}$ is strictly increasing. Therefore, by (32), we have

$$
\frac{5}{4}=u_{1} \leq u_{n}<\lim _{n \rightarrow \infty} u_{n}=\frac{4}{\pi}
$$

That is, inequalities (28) holds. This ends the proof of Lemma 7.

According to the theory of mathematical analysis, we have Lemma 8.

Lemma 8 Let the sequence $\left\{u_{n}\right\}_{n=1}^{\infty} \subset \mathbb{R}$ be convergent, and let $\lim _{n \rightarrow \infty} u_{n}=\mu$. Then we have

$$
\lim _{N \rightarrow \infty} \frac{1}{N} \sum_{n=1}^{N} u_{n}=\mu
$$

Lemma 9 The inequality

$$
\frac{2}{\pi} \int_{0}^{\frac{\pi}{2}} \frac{1+\mathrm{e}^{2} \cos ^{2} \theta}{\left(1-\mathrm{e}^{2} \cos ^{2} \theta\right)^{3 / 2}} \mathrm{~d} \theta \leq 1+\mu \frac{\mathrm{e}^{2}}{1-\mathrm{e}^{2}}
$$

holds if and only if

$$
\mu \leq \frac{4}{\pi}
$$

where the $\mu$ is a positive constant.

Proof Assume that (35) holds, we prove (34) as follows.

By the Newton formula (16), we get

$$
\begin{aligned}
& \frac{1+\mathrm{e}^{2} \cos ^{2} \theta}{\left(1-\mathrm{e}^{2} \cos ^{2} \theta\right)^{3 / 2}} \\
& =2\left(1-\mathrm{e}^{2} \cos ^{2} \theta\right)^{-3 / 2}-\left(1-\mathrm{e}^{2} \cos ^{2} \theta\right)^{-1 / 2} \\
& =1+2 \sum_{n=1}^{\infty} \frac{1}{n !} \prod_{k=1}^{n}\left(-\frac{3}{2}+1-k\right)\left(-\mathrm{e}^{2} \cos ^{2} \theta\right)^{n} \\
& \quad-\sum_{n=1}^{\infty} \frac{1}{n !} \prod_{k=1}^{n}\left(-\frac{1}{2}+1-k\right)\left(-\mathrm{e}^{2} \cos ^{2} \theta\right)^{n} \\
& =1+2 \mathrm{e}^{2} \sum_{n=1}^{\infty} \frac{(2 n+1) ! !}{(2 n) ! !} \mathrm{e}^{2(n-1)} \cos ^{2 n} \theta \\
& \quad-\mathrm{e}^{2} \sum_{n=1}^{\infty} \frac{(2 n-1) ! !}{(2 n) ! !} \mathrm{e}^{2(n-1)} \cos ^{2 n} \theta
\end{aligned}
$$




$$
\begin{aligned}
& =1+\mathrm{e}^{2} \sum_{n=1}^{\infty} \frac{2(2 n+1) ! !-(2 n-1) ! !}{(2 n) ! !} \mathrm{e}^{2(n-1)} \cos ^{2 n} \theta \\
& =1+\mathrm{e}^{2} \sum_{n=1}^{\infty} \frac{(4 n+1)(2 n-1) ! !}{(2 n) ! !} \mathrm{e}^{2(n-1)} \cos ^{2 n} \theta,
\end{aligned}
$$

that is,

$$
\frac{1+\mathrm{e}^{2} \cos ^{2} \theta}{\left(1-\mathrm{e}^{2} \cos ^{2} \theta\right)^{3 / 2}}=1+\mathrm{e}^{2} \sum_{n=1}^{\infty} \frac{(4 n+1)(2 n-1) ! !}{(2 n) ! !} \mathrm{e}^{2(n-1)} \cos ^{2 n} \theta
$$

Since

$$
\prod_{k=1}^{n}\left(1-\frac{1}{4 k^{2}}\right)=(2 n+1)\left[\frac{(2 n-1) ! !}{(2 n) ! !}\right]^{2}
$$

by (36), (37), and Lemma 5, we get

$$
\begin{aligned}
& \frac{2}{\pi} \int_{0}^{\frac{\pi}{2}} \frac{1+\mathrm{e}^{2} \cos ^{2} \theta}{\left(1-\mathrm{e}^{2} \cos ^{2} \theta\right)^{3 / 2}} \mathrm{~d} \theta \\
& \quad=1+\mathrm{e}^{2} \sum_{n=1}^{\infty} \frac{(4 n+1)(2 n-1) ! !}{(2 n) ! !} \mathrm{e}^{2(n-1)}\left(\frac{2}{\pi} \int_{0}^{\frac{\pi}{2}} \cos ^{2 n} \theta \mathrm{d} \theta\right) \\
& =1+\mathrm{e}^{2} \sum_{n=1}^{\infty} \frac{(4 n+1)(2 n-1) ! !}{(2 n) ! !} \mathrm{e}^{2(n-1)} \frac{(2 n-1) ! !}{(2 n) ! !} \\
& \quad=1+\mathrm{e}^{2} \sum_{n=1}^{\infty} \frac{4 n+1}{2 n+1} \prod_{k=1}^{n}\left(1-\frac{1}{4 k^{2}}\right) \mathrm{e}^{2(n-1)}
\end{aligned}
$$

that is,

$$
\frac{2}{\pi} \int_{0}^{\frac{\pi}{2}} \frac{1+\mathrm{e}^{2} \cos ^{2} \theta}{\left(1-\mathrm{e}^{2} \cos ^{2} \theta\right)^{3 / 2}} \mathrm{~d} \theta=1+\mathrm{e}^{2} \sum_{n=1}^{\infty} \frac{4 n+1}{2 n+1} \prod_{k=1}^{n}\left(1-\frac{1}{4 k^{2}}\right) \mathrm{e}^{2(n-1)} .
$$

By (38), $0 \leq \mathrm{e}<1$, and Lemma 7, we get

$$
\begin{aligned}
& \frac{2}{\pi} \int_{0}^{\frac{\pi}{2}} \frac{1+\mathrm{e}^{2} \cos ^{2} \theta}{\left(1-\mathrm{e}^{2} \cos ^{2} \theta\right)^{3 / 2}} \mathrm{~d} \theta \\
& \quad=1+\mathrm{e}^{2} \sum_{n=1}^{\infty} \frac{4 n+1}{2 n+1} \prod_{k=1}^{n}\left(1-\frac{1}{4 k^{2}}\right) \mathrm{e}^{2(n-1)} \\
& \quad \leq 1+\frac{4}{\pi} \mathrm{e}^{2} \sum_{n=1}^{\infty} \mathrm{e}^{2(n-1)} \\
& \quad=1+\frac{4}{\pi} \frac{\mathrm{e}^{2}}{1-\mathrm{e}^{2}} \\
& \quad \leq 1+\mu \frac{\mathrm{e}^{2}}{1-\mathrm{e}^{2}}
\end{aligned}
$$

Hence inequality (34) is proved.

Next, assume that (34) holds, we prove (35) as follows. 
Let $0<\mathrm{e}<1$. According to (38), we see that inequality (34) can be rewritten as

$$
1+\mathrm{e}^{2} \sum_{n=1}^{\infty} \frac{4 n+1}{2 n+1} \prod_{k=1}^{n}\left(1-\frac{1}{4 k^{2}}\right) \mathrm{e}^{2(n-1)} \leq 1+\mu \mathrm{e}^{2} \sum_{n=1}^{\infty} \mathrm{e}^{2(n-1)} .
$$

Hence

$$
\begin{aligned}
\mu & \geq \frac{\sum_{n=1}^{\infty} \frac{4 n+1}{2 n+1} \prod_{k=1}^{n}\left(1-\frac{1}{4 k^{2}}\right) \mathrm{e}^{2(n-1)}}{\sum_{n=1}^{\infty} \mathrm{e}^{2(n-1)}} \\
& =\frac{\lim _{N \rightarrow \infty} \sum_{n=1}^{N} \frac{4 n+1}{2 n+1} \prod_{k=1}^{n}\left(1-\frac{1}{4 k^{2}}\right) \mathrm{e}^{2(n-1)}}{\lim _{N \rightarrow \infty} \sum_{n=1}^{N} \mathrm{e}^{2(n-1)}} \\
& =\lim _{N \rightarrow \infty} \frac{\sum_{n=1}^{N} \frac{4 n+1}{2 n+1} \prod_{k=1}^{n}\left(1-\frac{1}{4 k^{2}}\right) \mathrm{e}^{2(n-1)}}{\sum_{n=1}^{N} \mathrm{e}^{2(n-1)}} \\
& =\lim _{N \rightarrow \infty} \frac{\sum_{n=1}^{N} u_{n} \mathrm{e}^{2(n-1)}}{\sum_{n=1}^{N} \mathrm{e}^{2(n-1)}},
\end{aligned}
$$

that is,

$$
\mu \geq \lim _{N \rightarrow \infty} \frac{\sum_{n=1}^{N} u_{n} \mathrm{e}^{2(n-1)}}{\sum_{n=1}^{N} \mathrm{e}^{2(n-1)}}, \quad \forall \mathrm{e} \in(0,1)
$$

where the sequence $\left\{u_{n}\right\}_{n=1}^{\infty}$ is defined by (31).

In (39), letting e $\rightarrow 1$, by (32), (39), and Lemma 8 , we get

$$
\mu \geq \lim _{\mathrm{e} \rightarrow 1} \lim _{N \rightarrow \infty} \frac{\sum_{n=1}^{N} u_{n} \mathrm{e}^{2(n-1)}}{\sum_{n=1}^{N} \mathrm{e}^{2(n-1)}}=\lim _{N \rightarrow \infty} \frac{1}{N} \sum_{n=1}^{N} u_{n}=\frac{4}{\pi} .
$$

This proves inequality (35). The proof of Lemma 9 is completed.

Lemma 10 The inequality

$$
\frac{2}{\pi} \int_{0}^{\frac{\pi}{2}} \frac{1+\mathrm{e}^{2} \cos ^{2} \theta}{\left(1-\mathrm{e}^{2} \cos ^{2} \theta\right)^{3 / 2}} \mathrm{~d} \theta \geq 1+v \frac{\mathrm{e}^{2}}{1-\mathrm{e}^{2}}
$$

holds if and only if

$$
v \leq \frac{5}{4}
$$

where the $v$ is a positive constant.

Proof Assume that (41) holds, we prove (40) as follows.

By (38), $0 \leq \mathrm{e}<1$, and Lemma 7, we have

$$
\begin{aligned}
& \frac{2}{\pi} \int_{0}^{\frac{\pi}{2}} \frac{1+\mathrm{e}^{2} \cos ^{2} \theta}{\left(1-\mathrm{e}^{2} \cos ^{2} \theta\right)^{3 / 2}} \mathrm{~d} \theta \\
& \quad=1+\mathrm{e}^{2} \sum_{n=1}^{\infty} \frac{4 n+1}{2 n+1} \prod_{k=1}^{n}\left(1-\frac{1}{4 k^{2}}\right) \mathrm{e}^{2(n-1)}
\end{aligned}
$$




$$
\begin{aligned}
& \geq 1+\frac{5}{4} \mathrm{e}^{2} \sum_{n=1}^{\infty} \mathrm{e}^{2(n-1)} \\
& =1+\frac{5}{4} \frac{\mathrm{e}^{2}}{1-\mathrm{e}^{2}} \\
& \geq 1+v \frac{\mathrm{e}^{2}}{1-\mathrm{e}^{2}}
\end{aligned}
$$

Hence inequality (40) is proved.

Next, assume that (40) holds, we prove (41) as follows.

Let $0<\mathrm{e}<1$. According to (38), we see that inequality (40) can be rewritten as

$$
1+\mathrm{e}^{2} \sum_{n=1}^{\infty} \frac{4 n+1}{2 n+1} \prod_{k=1}^{n}\left(1-\frac{1}{4 k^{2}}\right) \mathrm{e}^{2(n-1)} \geq 1+v \mathrm{e}^{2} \sum_{n=1}^{\infty} \mathrm{e}^{2(n-1)} .
$$

Hence

$$
v \leq \frac{\sum_{n=1}^{\infty} u_{n} \mathrm{e}^{2(n-1)}}{\sum_{n=1}^{\infty} \mathrm{e}^{2(n-1)}}, \quad \forall \mathrm{e} \in(0,1)
$$

where the sequence $\left\{u_{n}\right\}_{n=1}^{\infty}$ is defined by (31).

In (42), letting $\mathrm{e} \rightarrow 0$, we get

$$
v \leq u_{1}=\frac{5}{4}
$$

Hence inequality (41) is proved. This ends the proof of Lemma 10.

Lemma 11 The inequality

$$
\left[1-\left(1-\frac{2}{\pi}\right) \mathrm{e}^{2}\right]\left(1+\frac{5}{4} \frac{\mathrm{e}^{2}}{1-\mathrm{e}^{2}}\right) \geq 1+\eta \frac{\mathrm{e}^{2}}{1-\mathrm{e}^{2}}
$$

holds if and only if

$$
\eta \leq \frac{5}{2 \pi}
$$

where the $\eta$ is a positive constant.

Proof Let $0<\mathrm{e}<1$. Then inequality (43) can be rewritten as

$$
\eta \leq \frac{1-\mathrm{e}^{2}}{\mathrm{e}^{2}}\left\{\left[1-\left(1-\frac{2}{\pi}\right) \mathrm{e}^{2}\right]\left(1+\frac{5}{4} \frac{\mathrm{e}^{2}}{1-\mathrm{e}^{2}}\right)-1\right\}=\frac{2}{\pi}+\frac{1}{4}-\frac{1}{4}\left(1-\frac{2}{\pi}\right) \mathrm{e}^{2} .
$$

Hence inequality (43) holds if and only if

$$
\eta \leq \inf _{0<\mathrm{e}<1}\left\{\frac{2}{\pi}+\frac{1}{4}-\frac{1}{4}\left(1-\frac{2}{\pi}\right) \mathrm{e}^{2}\right\}=\frac{2}{\pi}+\frac{1}{4}-\frac{1}{4}\left(1-\frac{2}{\pi}\right)=\frac{5}{2 \pi} .
$$

That is, (43) holds if and only if (44) holds. This proves Lemma 11. 
Lemma 12 The inequality

$$
\sqrt{1-\frac{\mathrm{e}^{2}}{2}}\left(1+\frac{4}{\pi} \frac{\mathrm{e}^{2}}{1-\mathrm{e}^{2}}\right) \leq 1+\tau \frac{\mathrm{e}^{2}}{1-\mathrm{e}^{2}}
$$

holds if and only if

$$
\tau \geq \frac{16-\pi}{4 \pi},
$$

where $\tau$ is a positive constant.

Proof Let

$$
0<\mathrm{e}<1, \quad \varphi(\mathrm{e}) \triangleq-\frac{1+\left(\frac{4}{\pi}-1\right) \mathrm{e}^{2}}{2\left(\sqrt{1-\frac{\mathrm{e}^{2}}{2}}+1\right)}+\frac{4}{\pi} .
$$

Then inequality (45) can be rewritten as

$$
\tau \leq \frac{1-\mathrm{e}^{2}}{\mathrm{e}^{2}}\left[\sqrt{1-\frac{\mathrm{e}^{2}}{2}}\left(1+\frac{4}{\pi} \frac{\mathrm{e}^{2}}{1-\mathrm{e}^{2}}\right)-1\right]=-\frac{1+\left(\frac{4}{\pi}-1\right) \mathrm{e}^{2}}{2\left(\sqrt{1-\frac{\mathrm{e}^{2}}{2}}+1\right)}+\frac{4}{\pi}=\varphi(\mathrm{e}) .
$$

Since $3<\pi<4$, the functions

$$
1+\left(\frac{4}{\pi}-1\right) \mathrm{e}^{2} \text { and }\left[2\left(\sqrt{1-\frac{\mathrm{e}^{2}}{2}}+1\right)\right]^{-1}
$$

are strictly increasing, we see that the function $\varphi(\mathrm{e})$ is strictly decreasing. So we see that inequality (45) holds if and only if the inequality

$$
\tau \geq \sup _{0<\mathrm{e}<1}\{\varphi(\mathrm{e})\}=\varphi(0)=\frac{16-\pi}{4 \pi}
$$

holds. That is, (45) holds if and only if (46) holds. This ends the proof of Lemma 12.

Remark 1 According to Lemmas 4 and 6, we see that

$$
1-\left(1-\frac{2}{\pi}\right) \mathrm{e}^{2} \leq \frac{2}{\pi} \int_{0}^{\frac{\pi}{2}} \sqrt{1-\mathrm{e}^{2} \cos ^{2} \theta} \mathrm{d} \theta \leq \sqrt{1-\frac{1}{2} \mathrm{e}^{2}},
$$

where the coefficients $1-2 / \pi$ and $1 / 2$ of $\mathrm{e}^{2}$ are the best constants. Set

$$
\omega(t) \triangleq \sqrt{1-\frac{1}{2}} t-\left[1-\left(1-\frac{2}{\pi}\right) t\right], \quad t \in[0,1)
$$

then

$$
\begin{aligned}
\omega^{\prime}(t) & =-\frac{1}{2 \sqrt{4-2 t}}+1-\frac{2}{\pi}>\omega^{\prime}(1)=-\frac{1}{2 \sqrt{2}}+1-\frac{2}{\pi} \\
& =0.009826837039144887 \ldots>0 .
\end{aligned}
$$


Therefore, the error

$$
\begin{aligned}
\sqrt{1-\frac{1}{2} \mathrm{e}^{2}}-\left[1-\left(1-\frac{2}{\pi}\right) \mathrm{e}^{2}\right] & =\omega\left(\mathrm{e}^{2}\right)<\omega(1)=\frac{1}{\sqrt{2}}-\frac{2}{\pi} \\
& =0.07048700881896608 \ldots
\end{aligned}
$$

is very small.

Remark 2 According to Lemmas 9 and 10, we see that

$$
1+\frac{5}{4} \frac{\mathrm{e}^{2}}{1-\mathrm{e}^{2}} \leq \frac{2}{\pi} \int_{0}^{\frac{\pi}{2}} \frac{1+\mathrm{e}^{2} \cos ^{2} \theta}{\left(1-\mathrm{e}^{2} \cos ^{2} \theta\right)^{3 / 2}} \mathrm{~d} \theta \leq 1+\frac{4}{\pi} \frac{\mathrm{e}^{2}}{1-\mathrm{e}^{2}},
$$

where the coefficients $5 / 4$ and $4 / \pi$ of $\mathrm{e}^{2} /\left(1-\mathrm{e}^{2}\right)$ are the best constants, and the error

$$
\frac{4}{\pi}-\frac{5}{4}=0.023239544735162765 \ldots
$$

is also very small.

Remark 3 According to Lemma 11, we see that

$$
\left[1-\left(1-\frac{2}{\pi}\right) \mathrm{e}^{2}\right]\left(1+\frac{5}{4} \frac{\mathrm{e}^{2}}{1-\mathrm{e}^{2}}\right) \geq 1+\frac{5}{2 \pi} \frac{\mathrm{e}^{2}}{1-\mathrm{e}^{2}}
$$

where the coefficient $5 /(2 \pi)$ of $\mathrm{e}^{2} /\left(1-\mathrm{e}^{2}\right)$ is the best constant.

Remark 4 According to Lemma 12, we know that

$$
\sqrt{1-\frac{\mathrm{e}^{2}}{2}}\left(1+\frac{4}{\pi} \frac{\mathrm{e}^{2}}{1-\mathrm{e}^{2}}\right) \leq 1+\frac{16-\pi}{4 \pi} \frac{\mathrm{e}^{2}}{1-\mathrm{e}^{2}},
$$

where the coefficient $(16-\pi) /(4 \pi)$ of $\mathrm{e}^{2} /\left(1-\mathrm{e}^{2}\right)$ is also the best constant.

\section{Proof of Theorem 1}

Proof According to Lemma 3, and Remarks 1, 2, and 3, we have

$$
\begin{aligned}
\overline{\|\mathbf{F}(A, P)\|} & =\left(\frac{2 \pi}{|\Gamma|}\right)^{2}\left(\frac{2}{\pi} \int_{0}^{\frac{\pi}{2}} \sqrt{1-\mathrm{e}^{2} \cos ^{2} \theta} \mathrm{d} \theta\right)\left[\frac{2}{\pi} \int_{0}^{\frac{\pi}{2}} \frac{1+\mathrm{e}^{2} \cos ^{2} \theta}{\left(1-\mathrm{e}^{2} \cos ^{2} \theta\right)^{3 / 2}} \mathrm{~d} \theta\right] \\
& \geq\left(\frac{2 \pi}{|\Gamma|}\right)^{2}\left[1-\left(1-\frac{2}{\pi}\right) \mathrm{e}^{2}\right]\left(1+\frac{5}{4} \frac{\mathrm{e}^{2}}{1-\mathrm{e}^{2}}\right) \\
& \geq\left(\frac{2 \pi}{|\Gamma|}\right)^{2}\left(1+\frac{5}{2 \pi} \frac{\mathrm{e}^{2}}{1-\mathrm{e}^{2}}\right) .
\end{aligned}
$$

Hence the first inequality in (2) is proved. 
According to Lemma 3, and Remarks 1, 2, and 4, we have

$$
\begin{aligned}
\overline{\|\mathbf{F}(A, P)\|} & =\left(\frac{2 \pi}{|\Gamma|}\right)^{2}\left(\frac{2}{\pi} \int_{0}^{\frac{\pi}{2}} \sqrt{1-\mathrm{e}^{2} \cos ^{2} \theta} \mathrm{d} \theta\right)\left[\frac{2}{\pi} \int_{0}^{\frac{\pi}{2}} \frac{1+\mathrm{e}^{2} \cos ^{2} \theta}{\left(1-\mathrm{e}^{2} \cos ^{2} \theta\right)^{3 / 2}} \mathrm{~d} \theta\right] \\
& \leq\left(\frac{2 \pi}{|\Gamma|}\right)^{2} \sqrt{1-\frac{1}{2} \mathrm{e}^{2}}\left(1+\frac{4}{\pi} \frac{\mathrm{e}^{2}}{1-\mathrm{e}^{2}}\right) \\
& \leq\left(\frac{2 \pi}{|\Gamma|}\right)^{2}\left(1+\frac{16-\pi}{4 \pi} \frac{\mathrm{e}^{2}}{1-\mathrm{e}^{2}}\right) .
\end{aligned}
$$

Hence the second inequalities in (2) also hold.

Based on the above analysis, we see that equalities in (2) hold if and only if $\mathrm{e}=0$, that is, $\Gamma$ is a circle. This completes the proof of Theorem 1 .

\section{Mean temperature on a planet}

Let $S^{(2)}\{P, \Gamma\}$ be a centered surround system, where $\Gamma$ is an ellipse and $P$ is one of the foci of the ellipse. Then we may think that of $A \in \Gamma$ as a planet and of $P$ as the Sun, and of the ellipse $\Gamma$ as the motion trajectory of the planet. Assume that the radiation energy of the Sun $P$ to the planet $A$ is 1 , then, according to the optical laws, the radiant energy received by the planet $A$ is

$$
C\|\mathbf{F}(A, P)\|=\frac{C}{\|A-P\|^{2}},
$$

where $C>0$ is a constant of the radiation energy, and we can measure this constant $C$ by means of the physical methods.

As everyone knows, the radiant energy $C\|\mathbf{F}(A, P)\|$ is important to us. Since the rain and the air humidity are related to the radiation energy, we might think that there exist two constants $C_{*}, C^{*}>0$, such that $C_{*} \overline{\|\mathbf{F}(A, P)\|}$ is the mean air humidity and $C^{*} \overline{\|\mathbf{F}(A, P)\|}$ is the mean temperature on the Earth in a year. Therefore, Theorem 1 can be used to estimate the mean air humidity and the mean temperature on the Earth in a year.

Suppose that the planet $A$ is regarded as a particle, and the temperature on the planet $A$ at a certain moment is $T=T(A)$, and the mean temperature on the planet is $\bar{T}$. Then, based on the above analysis, there exists a constant $C^{*}>0$ such that

$$
T: \Gamma \rightarrow(0, \infty), \quad T(A)=C^{*}\|\mathbf{F}(A, P)\|
$$

and we can measure the constant $C^{*}$ by means of some tests. Without loss of generality, here we assume that $C^{*}=1$. Then, by (51), we have

$$
\bar{T}=\overline{\|\mathbf{F}(A, P)\|} .
$$

We remark here that, if the above planet is regarded as a sphere, then the point $A$ will be regarded as the center of the sphere, and the $T=T(A)$ will be regarded as the maximum temperature on the planet at the moment. In addition, $T$ can also be regarded as the mean of the air humidity on the Earth at a certain moment. 
According to Theorem 1 and (52), we have

$$
\left(1+\frac{5}{2 \pi} \frac{\mathrm{e}^{2}}{1-\mathrm{e}^{2}}\right)\left(\frac{2 \pi}{|\Gamma|}\right)^{2} \leq \bar{T} \leq\left(1+\frac{16-\pi}{4 \pi} \frac{\mathrm{e}^{2}}{1-\mathrm{e}^{2}}\right)\left(\frac{2 \pi}{|\Gamma|}\right)^{2} .
$$

By (53), we see that there exists a real function $\tau(\mathrm{e})$ such that

$$
\bar{T}=\overline{\|\mathbf{F}(A, P)\|}=\left(1+\tau(\mathrm{e}) \frac{\mathrm{e}^{2}}{1-\mathrm{e}^{2}}\right)\left(\frac{2 \pi}{|\Gamma|}\right)^{2},
$$

where

$$
0.7957747154594768 \ldots=\frac{5}{2 \pi} \leq \tau(\mathrm{e}) \leq \frac{16-\pi}{4 \pi}=1.0232395447351628 \ldots .
$$

Hence

$$
\tau(\mathrm{e}) \approx \frac{1}{2}\left(\frac{5}{2 \pi}+\frac{16-\pi}{4 \pi}\right)=\frac{26-\pi}{8 \pi}=0.9095071300973198 \ldots, \quad \forall \mathrm{e} \in(0,1) .
$$

According to (54) and (56), we get the following approximate mean temperature formula:

$$
\bar{T} \approx\left(1+0.9095071300973198 \ldots \times \frac{\mathrm{e}^{2}}{1-\mathrm{e}^{2}}\right)\left(\frac{2 \pi}{|\Gamma|}\right)^{2}, \quad \forall \mathrm{e} \in(0,1) .
$$

According to (57), we know that if the eccentricity e $\in(0,1)$ is very small, then the mean temperature $\bar{T}$ on the planet is also small. Conversely, if the eccentricity e $\in(0,1)$ is very large, then the mean temperature $\bar{T}$ on the planet is also very large. In particular, we have

$$
\lim _{\mathrm{e} \rightarrow 0} \bar{T}=\left(\frac{2 \pi}{|\Gamma|}\right)^{2} \text { and } \quad \lim _{\mathrm{e} \rightarrow 1} \bar{T}=\infty .
$$

This is the significance of Theorem 1 in the temperature research.

Suppose that the ellipses $\Gamma$ and $\Gamma_{*}$ are the motion trajectories of two planets, and the Sun $P$ is one of the foci of the two ellipses; $\mathrm{e}$ and $\mathrm{e}_{*}$ are the eccentricities of the two ellipses, respectively. Then, by inequality (53), we have

$$
\left(1+\frac{5}{2 \pi} \frac{\mathrm{e}^{2}}{1-\mathrm{e}^{2}}\right)\left(\frac{2 \pi}{|\Gamma|}\right)^{2}>\left(1+\frac{16-\pi}{4 \pi} \frac{\mathrm{e}_{*}^{2}}{1-\mathrm{e}_{*}^{2}}\right)\left(\frac{2 \pi}{\left|\Gamma_{*}\right|}\right)^{2} \Rightarrow \bar{T}>\overline{T^{*}},
$$

where $\bar{T}$ and $\overline{T^{*}}$ are the mean temperatures on the two planets, respectively. This is also the significance of Theorem 1 in the temperature research.

\section{Conclusions}

In this paper, we establish the gravity inequalities in the centered surround system $S^{(2)}\{P, \Gamma\}$, which are both an improvement and an expansion of inequality (3), where the $\Gamma$ is an ellipse and $P$ is one of the foci of the ellipse. We also demonstrate the applications of the inequalities in the temperature research on a planet, and we obtain an approximate 
mean temperature formula; we illustrate the significance of the formula in the temperature research on a planet.

The theoretical significance of this paper is to establish the geometric and physics theories on satellite motion, and the application value is to estimate the mean air humidity and the mean temperature on the Earth in a year. Large pieces of analysis, geometry, physics, and inequality theories are used in this paper, especially the mathematical analysis, and the series [17] is the crucial one.

Competing interests

The authors declare that they have no competing interests.

\section{Authors' contributions}

All authors contributed equally and significantly in this paper. All authors read and approved the final manuscript.

\section{Author details}

${ }^{1}$ Institute of Mathematical Inequalities and Applications, Chengdu University, Chengdu, Sichuan 610106, P.R. China.

${ }^{2}$ School of Information Engineering, Nanjing Xiaozhuang University, Nanjing, Jiangsu 211171, P.R. China. ${ }^{3}$ Department of Mathematics and Computer Science, Longyan University, Longyan, Fujian 364012, P.R. China.

\section{Acknowledgements}

The authors would like to acknowledge the support from the National Natural Science Foundation of China (No. 11161024 and No. 61309015), and the Natural Science Foundation of Fujian Province of China (No. 2016J01023), and the Natural Science Foundation of Sichuan Province Technology Department (No. 2014SZ0107).

Received: 12 June 2016 Accepted: 4 October 2016 Published online: 24 October 2016

\section{References}

1. Bullen, PS, Mitrinović, PS, Vasić, PM: Means and Their Inequalities. Reidel, Dordrecht (1988)

2. Wen, JJ, Gao, CB: Geometric inequalities involving the central distance of the centered 2-surround system. Acta Math. Sin. 51(4), 815-832 (2008) (in Chinese)

3. Gao, CB, Wen, JJ: Theory of surround system and associated inequalities. Comput. Math. Appl. 63, 1621-1640 (2012)

4. Gao, CB, Wen, JJ: Theories and inequalities on the satellite system. ISRN Math. Anal. 2011, Article ID 909261 (2011)

5. Wen, JJ, Yan, J, Wu, SH: Isoperimetric inequalities in surround system and space science. J. Inequal. Appl. 2016, 74 (2016)

6. Della Pietra, F, Gavitone, N: The relative isoperimetric inequality: the anisotropic case. J. Convex Anal. 20(1), 157-180 (2013)

7. Chung, FRK, Tetali, P: Isoperimetric inequalities for cartesian products of graphs. Comb. Probab. Comput. 7, 141-148 (1998)

8. King-Hele, D: Theory of Satellite Orbits in an Atmosphere. Butterworths, London (1964)

9. Gao, CB, Wen, JJ: A dimensionality reduction principle on the optimization of function. J. Math. Inequal. 7(3), 357-375 (2013)

10. Wen, JJ, Han, TY, Cheng, SS: Inequalities involving Dresher variance mean. J. Inequal. Appl. 2013, 366 (2013)

11. Yang, ZH, Chu, YM, Zhang, W: Accurate approximations for the complete elliptic integral of the second kind. J. Math. Anal. Appl. 438(2), 875-888 (2016)

12. Wen, JJ, Wang, WL: The inequalities involving generalized interpolation polynomial. Comput. Math. Appl. 56(4), 1045-1058 (2008)

13. Wen, JJ, Yuan, J, Yuan, SF: An optimal version of an inequality involving the third symmetric means. Proc. Indian Acad. Sci. Math. Sci. 118(4), 505-516 (2008)

14. Wen, JJ, Wang, WL: The optimization for the inequalities of power means. J. Inequal. Appl. 2006, Article ID 46782 (2006)

15. Wen, JJ, Wang, WL: Chebyshev type inequalities involving permanents and their applications. Linear Algebra Appl. 422(1), 295-303 (2007)

16. Wen, JJ, Pečarić, JE, Tian, YH: Weak monotonicity and Chebyshev type inequality. Math. Inequal. Appl. 18(1), 217-231 (2015)

17. Wen, JJ, Han, TY, Gao, CB: Convergence tests on constant Dirichlet series. Comput. Math. Appl. 62(9), $3472-3489$ (2011) 